\title{
Endothelial Dysfunction, a Marker of Atherosclerosis, Is Independent of Metabolic Syndrome in NAFLD Patients
}

\author{
Jimmy Narayan $\left(\mathbb{D},{ }^{1}\right.$ Haribhakti Seba Das $\mathbb{D}^{2},{ }^{2}$ Preetam Nath $\mathbb{D},{ }^{3}$ Ayaskanta Singh $\mathbb{D}^{1},{ }^{1}$ \\ Debakanta Mishra $\mathbb{D}^{1},{ }^{1}$ Pradeep Kumar Padhi $\mathbb{D}^{2},{ }^{2}$ and Shivaram Prasad Singh $\mathbb{D}^{2}$ \\ ${ }^{1}$ Department of Gastroenterology, IMS \& SUM Hospital, SOA University, Bhubaneswar, Odisha 751003, India \\ ${ }^{2}$ Department of Gastroenterology, SCB Medical College \& Hospital, Cuttack, Odisha 753001, India \\ ${ }^{3}$ Department of Gastroenterology, KIMS Hospital, KIIT University, Bhubaneswar, Odisha 751016, India
}

Correspondence should be addressed to Shivaram Prasad Singh; scb_gastro_dept@hotmail.com

Received 8 May 2020; Revised 4 July 2020; Accepted 6 July 2020; Published 17 July 2020

Academic Editor: Pierluigi Toniutto

Copyright ( 2020 Jimmy Narayan et al. This is an open access article distributed under the Creative Commons Attribution License, which permits unrestricted use, distribution, and reproduction in any medium, provided the original work is properly cited.

\begin{abstract}
Background. The study was designed to assess cardiovascular risk factors flow-mediated dilatation \% (FMD\%) and carotid intima-media thickness (CIMT) in NAFLD. Methods. 126 NAFLD subjects and 31 chronic hepatitis B (CHB) controls were studied. Measuring carotid intima-media thickness (CIMT) and the flow-mediated dilatation \% (FMD\%) by brachial artery Doppler ultrasound were used to assess atherosclerosis. The risk of cardiac events at 10 years (ROCE 10) was estimated by the Prospective Cardiovascular Munster Study (PROCAM) score. Results. 58 of 126 NAFLD have coexistent metabolic syndrome. Mean CIMT was $0.73 \pm 0.041 \mathrm{~mm}$ among NAFLD with MS, $0.66 \pm 0.016 \mathrm{~mm}$ among NAFLD without MS, and $0.66 \pm 0.037$ in controls CHB patients. FMD\% in NAFLD with MS was $10.43 \pm 3.134 \%$, but was $8.56 \pm 3.581 \%$ in NAFLD without MS and $17.78 \pm 6.051 \%$ in controls. PROCAM score of NAFLD with MS was $46.95 \pm 6.509$ while in NAFLD without MS was 38.2 \pm 3.738 . Controls had a PROCAM score of $38.13 \pm 5.755$. ROCE 10 in NAFLD with MS was $13.64 \pm 8.568$ while NAFLD without MS was $5.55 \pm 1.949$. Controls have a ROCE 10 of $5.95 \pm 3.973$. Post hoc analysis showed CIMT was dependent upon MS while FMD\% was different between all subgroups hence independent of metabolic syndrome. Conclusion. The markers of endothelial dysfunction are significantly higher in patients with NAFLD than controls.
\end{abstract}

\section{Introduction}

Nonalcoholic fatty liver disease (NAFLD) includes steatosis to steatohepatitis (NASH) [1]. NASH can progress on to cirrhosis and rarely to hepatocellular carcinoma (HCC) [2-4]. Moreover, NAFLD is one of the most common liver disorders in both developed and developing nations. Prevalence of NAFLD is estimated to be $15-35 \%$ in western countries [5] while it is 8-40\% in Asian countries [6-9].

NAFLD, obesity, type 2 diabetes mellitus (T2DM), and dyslipidemia frequently coexist. NAFLD is now considered a part of the spectrum of metabolic syndrome (MS). Increased risk for cardiovascular disease is associated with NAFLD. Patients with MS were approximately 1.5-2 times more likely to develop coronary artery disease (CAD) than the controls as shown in the $3^{\text {rd }}$ National Health and Nutrition Examination Survey, and Atherosclerosis Risk in Communities (ARIC) study [10]. Carotid intima-media thickness (CIMT) and endothelial dysfunction studied by flow-mediated vasodilatation (FMD) are noninvasive methods to assess cardiovascular risk factors and atherosclerosis [11]. In India, limited literature is available to show a significant association between these two. Western data have demonstrated the association between increased CIMT and NAFLD. Some had predicted the risk of atherosclerosis and cardiovascular disease to be independent of MS [12-14].

The Prospective Cardiovascular Munster Study (PROCAM) score [15], Adult Treatment Panel III (ATP III) [16], or Framingham score can predict the risk of cardiovascular disease.

The aim of the study was to evaluate the prevalence of atherosclerosis by measuring the CIMT and flow-mediated vasodilation (FMD) in Indian patients with incidentally detected NAFLD and predicting the risk of cardiovascular 
disease by using the PROCAM score in NAFLD patients and its association with metabolic syndrome (MS).

\section{Material and Methods}

Single-center, case-control study was conducted in the Department of Gastroenterology, S.C.B. Medical College and Hospital, Cuttack, between January 2014 and December 2015.

NAFLD patients attending Gastroenterology OPD, SCB Medical College, Cuttack, were taken as cases. The diagnosis of NAFLD was made on the basis of ultrasonography. Cases fulfilling fatty liver definition criteria which were defined according to the American Gastroenterology Association are as follows: an increase in hepatic echogenicity taking renal echogenicity as a reference, the presence of enhancement, and lack of differentiation in periportal intensity and the vesicular wall due to great hyperechogenicity of the parenchyma.

Controls were taken as patients of chronic hepatitis B with persistent/intermittent elevation in the levels of serum transaminase level (ALT/AST) greater than the upper limit of normal (ULN) for at least 6 months with $>6$ months of HBsAg positivity.

Exclusion criteria were patient with alcohol intake of $\geq 20$ g/d positive antibodies to hepatitis $\mathrm{C}$ virus (anti-HCV), positive autoimmune markers, abnormal iron profile drug usages such as corticosteroids, methotrexate or high-dose estrogens, and clinical or imaging features of cirrhosis of the liver.

All the subjects were explained thoroughly about the study. Those who signed informed consent were included in the study. Systemic examination was done. Body mass index (BMI), weight, waist circumference (WC), and hip circumference (HC) were measured in all patients. Complete blood count and routine biochemical investigations were performed in all subjects. The serum insulin level was assessed using the electrochemiluminescence method. IR was derived from FBG and plasma insulin (fasting insulin $(\mu \mathrm{U} / \mathrm{mL}) \times \mathrm{FBG}(\mathrm{mg} / \mathrm{dL})) / 405)$ and measured as HOMA-IR value [17]. For assessing risk cardiovascular factors, electrocardiography (ECG) was done. Carotid intima-media thickness (CIMT) was assessed using a highresolution B mode ultrasonography system (Phillips HD 11XE) with 7-12 MegaHertz transducer of both the right and left common carotid arteries at 3 points-base, midjunction, and just before bifurcation. The IMT is measured as the distance from the leading edge of the first echogenic line to the second echogenic line. The first echogenic line represents a luminal intimal interface while the second line represents the upper layer of intimal adventitia. IMTs were determined at the side of the greatest thickness and at two points, $1 \mathrm{~cm}$ upstream and $1 \mathrm{~cm}$ downstream. Six IMT measurements were done, and the mean was noted. For measuring brachial artery flow-mediated dilatation (FMD), rest and after reactive hyperemia diameter of the right brachial artery was measured. Tourniquet placed around the forearm to a pressure of $250 \mathrm{mmHg}$ for 4 minutes and 30 seconds followed by a release increase flow was studied. The arterial diameter was measured at a fixed distance from an anatomical marker at rest and at 40,60, and 80 seconds after the cuff release during systole. FMD $\%$ is calculated as $\mathrm{FMD} \%=$ $\mathrm{BADAV}-\mathrm{BADB} / \mathrm{BADB}$, where $\mathrm{BADB}$ is the brachial artery diameter at rest and postischemia is the BADAV. The risk of cardiac events at 10 years (ROCE 10) was estimated by the Prospective Cardiovascular Munster Study (PROCAM) score. The PROCAM score includes age, blood pressure, diabetes, cigarette smoking, total and low-density cholesterol, TGs, and family history of myocardial infarction.

2.1. Definitions. National Cholesterol Education ProgramAdult Treatment Panel III (NCEP-ATP III) guidelines $[18,19]$ were used to define hypercholesterolemia. American Diabetes Association (ADA) criteria were used to define diabetes mellitus [20]. Normal ALT and AST values were taken as 0-40IU/L. Hypertension was defined as blood pressure $\geq 140 / 90 \mathrm{mmHg}$ or treatment with antihypertensive drugs. Having a normal fasting insulin level of 6-27 $\mu \mathrm{U} / \mathrm{mL}$ and a homeostasis model assessment of insulin resistance (HOMA-IR) value of $\geq 2.00$ was taken as insulin resistance [21].

Obesity guidelines based on western populations is inaccurate for Asian individual; hence, the Asian Indians cut off was used in our study [22]. Overweight and obesity cut off was $\mathrm{BMI} \geq 23 \mathrm{~kg} / \mathrm{m}^{2}$ and $\geq 25 \mathrm{~kg} / \mathrm{m}^{2}$, respectively. Metabolic syndrome diagnostic criteria were defined according to 2001 modified NCEP-ATP III-2 guidelines, which include any three of the following:

(1) Abdominal obesity: men $\geq 90 \mathrm{~cm}$, women $\geq 80 \mathrm{~cm}$

(2) Serum $\mathrm{TG} \geq 150 \mathrm{mg} / \mathrm{dL}$

(3) HDL-C $<40 \mathrm{mg} / \mathrm{dL}$ (male), $<50 \mathrm{mg} / \mathrm{dL}$ (female)

(4) Fasting blood glucose level $\geq 100 \mathrm{mg} / \mathrm{dL}$

(5) Blood pressure $\geq 130 / \geq 85 \mathrm{mmHg}$

Statistical analysis was performed using SPSS 15.0. Student's $t$-test and Mann-Whitney $U$ test were used for parametric and nonparametric continuous data, respectively, whereas chi-square test was applied for the categorical data. The strength of the correlation between quantitative variables was calculated by Pearson correlation coefficient, and Spearman correlation analysis was used for that of categorical variables. The final outcome variables were studied (NAFLD patients with MS, without MS and controls) using the Kruskal-Wallis test with subsequent pair-wise post hoc analysis. " $p$ " value of $<0.05$ was defined as statistically significant.

\section{Results}

A total of 126 NAFLD subjects as cases and 31 chronic hepatitis $\mathrm{B}(\mathrm{CHB})$ patients as controls were enrolled in this study. NAFLD with metabolic syndrome were 58 subjects while the rest were NAFLD without metabolic syndrome. Among the controls (CHB), 19 (60\%) were HBeAg negative, and the remaining $12(40 \%)$ were $\mathrm{HBeAg}$ positive with a mean HBV DNA of $14.7 \times 10^{4}$; standard deviation $=21.7 \times 10$ [3]. 
The male-female ratio was $2: 1$ among controls and $2.5: 1$ among cases; the gender difference between the two groups was comparable $(p=0.66)$. Baseline anthropometrical and biochemical data comparison between NAFLD and controls (CHB) is given in Table 1 .

Baseline comparison between NAFLD with and without metabolic syndrome is depicted in Table 2, which showed that NAFLD with metabolic syndrome has higher BMI, waist, waist/hip, waist/height ratio, and higher systolic blood pressure as compared to NAFLD without metabolic syndrome. Furthermore, NAFLD subjects with metabolic syndrome had significantly higher fasting blood sugar, higher HOMA IR values, higher serum triglycerides, and lower HDL cholesterol than those without, whereas total, LDL, and VLDL cholesterol was comparable between these two groups. Besides, the LFT profile was comparable in both groups except for higher alkaline phosphatase and gammaglutamyl peptidase in subjects with metabolic syndrome.

Mean carotid intima media thickness (right) was $0.73+$ $0.046 \mathrm{~mm}$ among NAFLD with MS, $0.66+0.022 \mathrm{~mm}$ among NAFLD without MS, and $0.64+0.043$ among controls chronic hepatitis B patients. Mean CIMT (left) was $0.74+$ $0.04 \mathrm{~mm}$ among NAFLD with MS, $0.67+0.012 \mathrm{~mm}$ among NAFLD without MS, and $0.67+0.044$ among controls chronic hepatitis B patients. Mean CIMT (mean) is $0.73+$ $0.041 \mathrm{~mm}$ among NAFLD with MS, $0.66+0.016 \mathrm{~mm}$ among NAFLD without MS, and $0.66+0.037$ among controls chronic hepatitis B patients. ANOVA showed significant difference between the groups and within the group for carotid intima media thickness (CIMT) (Table 3). Brachial artery dimension (BADB) at baseline is $3.92+0.228 \mathrm{~mm}$ among NAFLD with MS, $3.87+0.205 \mathrm{~mm}$ in NAFLD without MS, and $3.73+0.158 \mathrm{~mm}$ among chronic hepatitis $\mathrm{B}$ controls.

The markers of endothelial dysfunction such as carotid intima-media thickness (CIMT), brachial artery dimension at baseline (BADB), brachial artery dimension average after vasodilation (BADAV), flow-mediated vasodilation (FMD), PROCAM, and ROCE 10 score were compared in Table 3 and revealed a significant difference among the NAFLD with and without metabolic syndrome and the controls $(p<0.001$, calculated by ANOVA). The scores were worse in patients with NAFLD and metabolic syndrome than those without and controls.

Post hoc analysis showed that carotid intima-media thickness is dependent upon metabolic syndrome while flowmediated vasodilation is different between all subgroups, hence independent of metabolic syndrome (Table 4). A strong inverse correlation was found between the PROCAM score and FMD\% ( $p<0.0001)$; also, a positive correlation was found between the PROCAM score and CIMT. ROCE10 was found to have a strong positive correlation with the PROCAM score $(p<0.0001)$ and CIMT (Table 5).

\section{Discussion}

The association between NAFLD and metabolic syndrome is previously studied sparsely $[23,24]$. Some considered it as a part of metabolic syndrome $[25,26]$. In the present study, we studied atherosclerosis as measured by CIMT between
TABLE 1: Baseline anthropometrical and biochemical comparison between NAFLD and controls (chronic hepatitis B (CHB)).

\begin{tabular}{|c|c|c|c|}
\hline Parameters & $\begin{array}{l}\text { NAFLD } \\
(n=126)\end{array}$ & $\mathrm{CHB}(n=31)$ & $p$ value \\
\hline Age & $45.78 \pm 11.67$ & $46.55 \pm 11.89$ & 0.74 \\
\hline BMI & $28.97 \pm 6.33$ & $27.02 \pm 3.63$ & 0.10 \\
\hline Waist circumference & $100.83 \pm 12.81$ & $94.68 \pm 8.32$ & 0.01 \\
\hline Waist/hip ratio & $0.99 \pm 0.08$ & $0.97 \pm 0.08$ & 0.27 \\
\hline Waist/height ratio & $0.62 \pm 0.1$ & $0.61 \pm 0.06$ & 0.23 \\
\hline SBP & $128.65 \pm 21.05$ & $126.9 \pm 9.09$ & 0.65 \\
\hline DBP & $81.5 \pm 11.6$ & $82.1 \pm 4.7$ & 0.77 \\
\hline Total bilirubin & $1.15 \pm 3.03$ & $0.79 \pm 0.25$ & 0.51 \\
\hline AST & $34.02 \pm 18.32$ & $44.23 \pm 20.29$ & 0.08 \\
\hline ALT & $47.71 \pm 32.89$ & $54.7 \pm 31.93$ & 0.07 \\
\hline Alkaline phosphatase & $180.48 \pm 71.24$ & $220.42 \pm 64.75$ & 0.005 \\
\hline Total protein & $7.9 \pm 2.46$ & $7.36 \pm 0.56$ & 0.45 \\
\hline Albumin & $4.21 \pm 0.28$ & $4.19 \pm 0.29$ & 0.73 \\
\hline Globulin & $3.75 \pm 0.66$ & $3.16 \pm 0.55$ & 0.42 \\
\hline GGT & $34.56 \pm 22.98$ & $27.92 \pm 26.58$ & 0.17 \\
\hline Insulin & $11.18 \pm 7.13$ & $8.14 \pm 3.75$ & 0.02 \\
\hline HOMA IR & $3.17 \pm 4.29$ & $2.07 \pm 1.39$ & 0.01 \\
\hline FBS & $112.8 \pm 44.7$ & $104.6 \pm 17.14$ & 0.32 \\
\hline 2hour PPBS & $171.8 \pm 83.82$ & $141.2 \pm 42.11$ & 0.05 \\
\hline Triglycerides & $176.8 \pm 86.73$ & $183.4 \pm 76.41$ & 0.70 \\
\hline Total cholesterol (TC) & $197.4 \pm 47.19$ & $213.3 \pm 36.3$ & 0.08 \\
\hline HDL & $47.68 \pm 18.43$ & $44.65 \pm 7.83$ & 0.37 \\
\hline LDL & $118.8 \pm 33.1$ & $131.9 \pm 28.27$ & 0.04 \\
\hline VLDL & $35.9 \pm 19.05$ & $38.9 \pm 15.68$ & 0.41 \\
\hline LDL/HDL & $2.82 \pm 0.89$ & $2.82 \pm 0.48$ & 0.99 \\
\hline TC/HDL & $4.44 \pm 1.06$ & $4.77 \pm 0.69$ & 0.29 \\
\hline
\end{tabular}

patients of NAFLD and chronic hepatitis B controls. Targher et al. found similar results in NAFLD with CIMT $(1.14 \pm 0.20$ vs. $0.82 \pm 0.12 \mathrm{~mm} ; p<0.001)$ values higher than controls [12], and MS components were more frequent in NAFLD but the difference in CIMT observed between the groups was weakened following adjustment for individual metabolic syndrome components. Volzke et al. [27] postulated higher CIMT and more carotid plaques in fatty liver patients than controls (plaque prevalence rate $76.8 \%$ vs. $66.6 \% ; p<$ 0.001 ). Brea et al. also reported higher CIMT values in NAFLD subjects which were independent of MS even after adjustment and logistic regression [28].

Duseja et al. found similar CIMT values $(0.70+0.11)$ among NAFLD patients as compared to our study [3]. Also, in our study, no significant CIMT difference between NAFLD without metabolic syndrome and controls was found replicating the previous study of metabolic syndrome being the prime contributor to atherosclerosis. 
TABle 2: Baseline clinical, anthropometrical, and biochemical comparison between NAFLD with MS and NAFLD without MS

\begin{tabular}{|c|c|c|c|}
\hline Parameters & $\begin{array}{l}\text { NAFLD with MS } \\
\quad(n=58)\end{array}$ & $\begin{array}{l}\text { NAFLD without MS } \\
\qquad(n=68)\end{array}$ & $\begin{array}{c}p \\
\text { value }\end{array}$ \\
\hline Age & $47.55 \pm 11.46$ & $44.26 \pm 11.71$ & 0.11 \\
\hline BMI & $33.78 \pm 4.86$ & $24.87 \pm 4.21$ & $<0.001$ \\
\hline $\begin{array}{l}\text { Waist } \\
\text { circumference }\end{array}$ & $113.24 \pm 4.69$ & $90.24 \pm 6.36$ & $<0.001$ \\
\hline Waist/hip ratio & $1.02 \pm 0.08$ & $0.96 \pm 0.06$ & $<0.001$ \\
\hline $\begin{array}{l}\text { Waist/height } \\
\text { ratio }\end{array}$ & $0.7 \pm 0.06$ & $0.56 \pm 0.08$ & $<0.001$ \\
\hline SBP & $134.5 \pm 22.59$ & $118.65 \pm 13.26$ & $<0.001$ \\
\hline DBP & $82.8 \pm 12.73$ & $79.4 \pm 9.28$ & 0.17 \\
\hline Total bilirubin & $0.74 \pm 0.23$ & $1.51 \pm 0.86$ & 0.16 \\
\hline AST & $32 \pm 11.3$ & $35.7 \pm 22.7$ & 0.25 \\
\hline $\begin{array}{l}\text { Alkaline } \\
\text { phosphatase }\end{array}$ & $204.1 \pm 69.7$ & $160.5 \pm 66.7$ & 0.001 \\
\hline Total protein & $7.19 \pm 0.73$ & $7.36 \pm 0.56$ & 0.125 \\
\hline Albumin & $4.18 \pm 0.23$ & $4.23 \pm 0.33$ & 0.358 \\
\hline Globulin & $3.03 \pm 0.69$ & $4.55 \pm 0.55$ & 0.42 \\
\hline GGT & $39.23 \pm 25.05$ & $29.96 \pm 19.91$ & 0.03 \\
\hline Insulin & $14.22 \pm 7.93$ & $8.03 \pm 4.39$ & $<0.001$ \\
\hline HOMA IR & $4.89 \pm 5.7$ & $1.71 \pm 1.42$ & $<0.001$ \\
\hline FBS & $126.59 \pm 59.62$ & $100.87 \pm 19.29$ & 0.002 \\
\hline 2hour PPBS & $197.4 \pm 98.3$ & $149.42 \pm 61.06$ & 0.001 \\
\hline Triglycerides & $194.62 \pm 103.4$ & $161.68 \pm 66.57$ & 0.033 \\
\hline $\begin{array}{l}\text { Total } \\
\text { cholesterol } \\
\text { (TC) }\end{array}$ & $197.9 \pm 45.59$ & $197 \pm 48.85$ & 0.913 \\
\hline HDL & $43.9 \pm 9.03$ & $51.68 \pm 23.01$ & 0.008 \\
\hline LDL & $117.0 \pm 36.93$ & $120.49 \pm 29.64$ & 0.558 \\
\hline VLDL & $39.21 \pm 19.24$ & $33.06 \pm 18.56$ & 0.073 \\
\hline LDL/HDL & $2 / 88 \pm 0.87$ & $2.78 \pm 0.87$ & 0.629 \\
\hline TC/HDL & $4.23 \pm 1.1$ & $4.68 \pm 0.96$ & 0.06 \\
\hline
\end{tabular}

In this study, NAFLD was associated with increased carotid IMT independently of other risk factors. Other factors of atherosclerosis may be playing an important role. Oxidative stress may play an important factor in the progression of NAFLD [29].

Brachial artery dimension at baseline and average dimension after vasodilation were higher among NAFLD as compared to chronic hepatitis B controls and, ANOVA analysis showed it to be a significant difference between NAFLD with and without metabolic syndrome. In our study, FMD\% taken as a predictor of early atherosclerosis and endothelial dysfunction was found to be significantly lower in the NAFLD group $(9.45 \pm 3.49 \%)$ than in controls $((17.78 \pm 3.49 \%) p<$ $0.0001)$. Villanova et al. also found similar results [13]. The FMD\% in their study was remarkably higher in fatty liver (9.93\%) as compared to NASH cases ((4.94\%) $p=0.01)$. But after adjustment of confounding factors, they suggested
TABLE 3: Carotid intima-media thickness (CIMT), brachial artery dimension at baseline (BADB), brachial artery dimension average after vasodilation (BADAV), flow-mediated vasodilation (FMD), PROCAM, and ROCE 10 score in NAFLD (with and without MS) and $\mathrm{CHB}$.

\begin{tabular}{lcccc}
\hline & $\begin{array}{c}\text { NAFLD with } \\
\text { MS }(n=58)\end{array}$ & $\begin{array}{c}\text { NAFLD } \\
\text { without MS } \\
(n=68)\end{array}$ & $\begin{array}{c}\text { CHB } \\
(n=31)\end{array}$ & $\begin{array}{c}p \\
\text { value* }\end{array}$ \\
\hline CMIT R & $0.73 \pm 0.05$ & $0.66 \pm 0.02$ & $0.64 \pm 0.04$ & $<0.001$ \\
CIMT L & $0.74 \pm 0.04$ & $0.67 \pm 0.01$ & $0.67 \pm 0.04$ & $<0.001$ \\
CIMT M & $0.73 \pm 0.04$ & $0.66 \pm 0.02$ & $0.66 \pm 0.04$ & $<0.001$ \\
BADB & $3.92 \pm 0.23$ & $3.87 \pm 0.21$ & $3.73 \pm 0.16$ & $<0.001$ \\
BADAV & $4.32 \pm 0.17$ & $4.20 \pm 0.18$ & $4.39 \pm 0.11$ & $<0.001$ \\
FMD & $10.43 \pm 3.13$ & $8.56 \pm 3.58$ & $17.78 \pm 6.05$ & $<0.001$ \\
PROCAM & $46.95 \pm 6.51$ & $38.20 \pm 3.74$ & $38.13 \pm 5.8$ & $<0.001$ \\
ROCE 10 & $13.64 \pm 8.57$ & $5.95 \pm 1.97$ & $5.55 \pm 1.94$ & $<0.001$ \\
\hline
\end{tabular}

${ }^{*}$ Calculated by ANOVA.

the role of MS rather than NAFLD as the cause. Duseja et al. found FMD\% among NAFLD group $(9.7 \pm 3.81 \%)$ different in comparison to controls $((17.03 \pm 3.39 \%) p<$ $0.0001)$, but no difference in FMD\% among patients of NAFLD with and without MS was found [3].

Thakur et al. [30] studied 40 nondiabetic subjects with NAFLD and 40 controls without NAFLD and measured atherosclerosis parameters and found similar results to our studies. NAFLD was found to be an independent predictor of CIMT and impaired FMD even after adjusting for different confounding factors.

Our study has interesting results while endothelial dysfunction, a marker of atherosclerosis and vascular instability, is independent of metabolic syndrome among fatty liver subjects supporting Thakur et al.'s study but carotid intimamedia thickness (CIMT), an advance marker of cardiovascular risk factors, is dependent upon metabolic syndrome supporting Duseja's group. It could be due to a smaller sample size in Duseja et al.'s study and also different pathophysiology of endothelial dysfunction and CIMT thickness. More insight into this difference needs study based on molecular pathogenesis changes like studies implicating NO.

Increased atherosclerosis increases the risk of future cardiovascular events and also correlate with the severity of coronary atherosclerosis.

In our study, we observed that cardiovascular risk factor predicting scores (PROCAM, ROCE 10) were higher among fatty liver subjects which were dependent on the presence of MS. Villanova et al. found that ROCE10 moderately increased $(p=0.045)$ in patients with fatty liver [13]. Duseja et al. showed similar results among north Indian cohorts which show PROCAM and ROCE 10 dependent upon metabolic syndrome [3]. PROCAM score was found higher in fatty liver $(27.50 \pm 13.32)$ as compared to controls $(20.10 \pm 7.75)(p<0.05)$. The PROCAM score of NAFLD and controls in our cohort is higher than the previous study which could be ascribed to the higher metabolic and cardiovascular risk factors in our group. Kessler et al. found a 
TABle 4: Post hoc analysis of carotid intima-media thickness (CIMT) and flow-mediated vasodilation (FMD).

\begin{tabular}{lccc}
\hline Variables & $\begin{array}{c}\text { NAFLD with MS } \\
(n=58)\end{array}$ & $\begin{array}{c}\text { NAFLD without MS } \\
(n=68)\end{array}$ & $\begin{array}{c}p \\
\text { value }^{*}\end{array}$ \\
\hline \multirow{4}{*}{ CIMT } & NAFLD without MS & NAFLD with MS & $<0.001$ \\
& NAFLD without MS & CHB & 0.976 \\
& NAFLD with MS & CHB & $<0.001$ \\
\multirow{4}{*}{ FMD } & NAFLD without MS & NAFLD with MS & 0.036 \\
& NAFLD without MS & CHB & $<0.001$ \\
& NAFLD with MS & CHB & $<0.001$ \\
\hline
\end{tabular}

TABLE 5: Correlation between PROCAM score, CIMT, ROCE10, and FMD.

\begin{tabular}{lccc}
\hline Variable 1 & Variable 2 & $p$ value $^{*}$ & Correlation \\
\hline PROCAM & CIMT M & $<0.001$ & Positive \\
PROCAM & FMD & $<0.001$ & Inverse \\
PROCAM & ROCE 10 & $<0.001$ & Positive \\
CIMT M & ROCE 10 & $<0.001$ & Positive \\
ROCE 10 & FMD & 0.168 & \\
\hline
\end{tabular}

higher prevalence of myocardial infarction (66\% and 50\% for women and men, respectively) in the fatty liver while comparing to the normal population [31]. A prospective study involving 1221 participants by Hamaguchi et al. found an increased incidence of cardiovascular disease (coronary heart disease, ischaemic stroke, and cerebral hemorrhage) in 231 patients with the fatty liver as compared to 990 normal population without NAFLD [32]. We found a strong $(p<0.001)$ inverse correlation between the PROCAM score and FMD\%. PROCAM score and CIMT show a positive correlation in fatty liver subjects. ROCE10 also shows a positive correlation $(p<0.0001)$ with the PROCAM score and CIMT. Duseja et al. have found similar results but they found a strong correlation between FMD and ROCE 10 which was absent in our study; probably larger sample size with more homogenous population would shed better light in this subject [3].

The practical implication of our study is that a reproducible assessment of cardiovascular risk factors which is the prime cause of mortality among NAFLD is studied.

Histopathology diagnosis for fatty liver diagnosis is a limitation of our study. In a recent study, NAFLD fibrosis score (NFS), as a marker of NAFLD, could identify patients at higher risk of CVD; this is a relevant finding [33]. Ultrasonography cannot identify fatty infiltration of the liver below $30 \%$ which makes liver biopsy as the gold standard to diagnose fatty liver but is not practical in this group. In our study, we have not assessed calorie intake, physical activity, sedentary lifestyle, and smoking habits which also have an impact on the cardiovascular risk factor.

\section{Data Availability}

Data are available and will be provided on request.

\section{Conflicts of Interest}

The authors declare that they have no conflicts of interest.

\section{Authors' Contributions}

Jimmy Narayan is responsible for the study design, data collection, data analysis, and writing; Haribhakti Seba Das for the study design, data collection, and data analysis; Preetam Nath for the data collection and data analysis; Ayaskanta Singh for the study design, data collection, data analysis, and writing; Debakanta Mishra for the study design, data collection, data analysis, and writing; Pradeep Kumar Padhi for the data collection and data analysis; and Shivaram Prasad Singh for the study design, data collection, data analysis, and writing of this paper.

\section{References}

[1] Y. Falck-Ytter, Z. M. Younossi, G. Marchesini, and A. J. McCullough, "Clinical features and natural history of nonalcoholic steatosis syndromes," Seminars in Liver Disease, vol. 21, no. 1, pp. 17-26, 2001.

[2] B. A. Neuschwander-Tetri and S. H. Caldwell, "Nonalcoholic steatohepatitis: summary of an AASLD single topic conference," Hepatology, vol. 37, no. 5, pp. 1202-1219, 2003.

[3] A. Duseja, B. Sharma, A. Kumar et al., "Nonalcoholic fatty liver in a developing country is responsible for significant liver disease," Hepatology, vol. 52, no. 6, pp. 2248-2249, 2010.

[4] A. Duseja, M. Nanda, A. Das, R. Das, A. Bhansali, and Y. Chawla, "Prevalence of obesity, diabetes mellitus and hyperlipidemia in patients with cryptogenic liver cirrhosis," Tropical Gastroenterology, vol. 25, no. 1, pp. 15-17, 2004.

[5] G. C. Farrell and C. Z. Larter, "Nonalcoholic fatty liver disease: from steatosis to cirrhosis," Hepatology, vol. 43, pp. S00-S112, 2006.

[6] S. P. Singh, S. Nayak, M. Swain et al., "Prevalence of nonalcoholic fatty liver disease in coastal eastern India: a preliminary ultrasonographic survey," Tropical Gastroenterology, vol. 25, no. 2, pp. 76-79, 2004.

[7] C. P. Day and O. F. W. James, "Steatohepatitis: A tale of two "hits"?,” Gastroenterology, vol. 114, no. 4, pp. 842-845, 1998.

[8] J. M. Hui, A. Hodge, G. C. Farrell, J. G. Kench, A. Kriketos, and J. George, "Beyond insulin resistance in NASH: TNF-alpha or adiponectin?," Hepatology, vol. 40, no. 1, pp. 46-54, 2004.

[9] D. Amarapurkar, P. Kamani, N. Patel et al., "Prevalence of non-alcoholic fatty liver disease: population-based study," Annals of Hepatology, vol. 6, no. 3, pp. 161-163, 2007.

[10] A. M. McNeill, W. D. Rosamond, C. J. Girman et al., "The metabolic syndrome and 11-year risk of incident cardiovascular disease in the atherosclerosis risk in communities study," Diabetes Care, vol. 28, no. 2, pp. 385-390, 2005.

[11] M. C. Corretti, T. J. Anderson, E. J. Benjamin et al., "Guidelines for the ultrasound assessment of endothelial-dependent flowmediated vasodilatation of the brachial artery: a report of the International Brachial Artery," Journal of the American College of Cardiology, vol. 39, no. 2, pp. 257-265, 2002.

[12] G. Targher, L. Bertolini, R. Padovani, L. Zenari, G. Zoppini, and G. Falezza, "Relation of nonalcoholic hepatic steatosis to early carotid atherosclerosis in healthy men: role of visceral 
fat accumulation," Diabetes Care, vol. 27, no. 10, pp. 24982500, 2004.

[13] N. Villanova, S. Moscatiello, S. Ramilli et al., "Endothelial dysfunction and cardiovascular risk profile in nonalcoholic fatty liver disease," Hepatology, vol. 42, no. 2, pp. 473-480, 2005.

[14] C. H. Tsai, T. C. Li, and C. C. Lin, "Metabolic syndrome as a risk factor for nonalcoholic fatty liver disease," Southern Medical Journal, vol. 101, no. 9, pp. 900-905, 2008.

[15] G. Assmann, P. Cullen, and H. Schulte, "Simple scoring scheme for calculating the risk of acute coronary events based on the 10-year follow-up of the prospective cardiovascular Munster (PROCAM) study," Circulation, vol. 105, no. 3, pp. 310-315, 2002.

[16] Expert panel on detection, evaluation, and treatment of high cholesterol in adults, "Executive Summary of the Third Report of the National Cholesterol Education Program (NCEP) Expert Panel on detection, evaluation, and treatment of high blood cholesterol in adults (Adult Treatment Panel III)," The Journal of the American Medical Association, vol. 285, no. 19, pp. 2486-2497, 2001.

[17] K. Kantartzis, C. Thamer, A. Peter et al., "High cardiorespiratory fitness is an independent predictor of the reduction in liver fat during a lifestyle intervention in non-alcoholic fatty liver disease," Gut, vol. 58, no. 9, pp. 1281-1288, 2009.

[18] M. W. Lorenz, H. S. Markus, M. L. Bots, M. Rosvall, and M. Sitzer, "Prediction of clinical cardiovascular events with carotid intima-media thickness: a systematic review and meta-analysis," Circulation, vol. 115, no. 4, pp. 459-467, 2007.

[19] T. Ohira, E. Shahar, H. Iso et al., "Carotid artery wall thickness and risk of stroke Subtypes," Stroke, vol. 42, no. 2, pp. 397-403, 2011.

[20] T. F. Lüscher and M. Barton, "Biology of the endothelium," Clinical Cardiology, vol. 20, pp. 3-10, 1997.

[21] R. F. Furchgott and J. V. Zawadzki, "The obligatory role of endothelial cells in the relaxation of arterial smooth muscle by acetylcholine," Nature, vol. 288, no. 5789, pp. 373-376, 1980.

[22] H. Lu, H. Liu, F. Hu, L. Zou, S. Luo, and L. Sun, "Independent association between nonalcoholic fatty liver disease and cardiovascular disease: a systematic review and meta-analysis," International Journal of Endocrinology, vol. 2013, Article ID 124958, 7 pages, 2013.

[23] A. Gastaldelli, K. Cusi, M. Pettiti et al., "Relationship between hepatic/visceral fat and hepatic insulin resistance in nondiabetic and type 2 diabetic subjects," Gastroenterology, vol. 133, no. 2, pp. 496-506, 2007.

[24] E. Fabbrini, B. S. Mohammed, F. Magkos, K. M. Korenblat, B. W. Patterson, and S. Klein, "Alterations in Adipose Tissue and Hepatic Lipid Kinetics in Obese Men and Women With Nonalcoholic Fatty Liver Disease," Gastroenterology, vol. 134, no. 2, pp. 424-431, 2008.

[25] E. Vanni, E. Bugianesi, A. Kotronen, S. De Minicis, H. Yki-Jarvinen, and G. Svegliati-Baroni, "From the metabolic syndrome to NAFLD or vice versa?," Digestive and Liver Disease, vol. 42, no. 5, pp. 320-330, 2010.

[26] A. Gastaldelli, "Fatty liver disease: the hepatic manifestation of metabolic syndrome," Hypertension Research, vol. 33, no. 6, pp. 546-547, 2010.

[27] H. Völzke, D. M. Robinson, V. Kleine et al., "Hepatic steatosis is associated with an increased risk of carotid atherosclerosis,"
World Journal of Gastroenterology, vol. 11, no. 12, pp. 18481853, 2005.

[28] A. Brea, D. Mosquera, E. Martín, A. Arizti, J. L. Cordero, and E. Ros, "Nonalcoholic fatty liver disease is associated with carotid Atherosclerosis," Arteriosclerosis, Thrombosis, and Vascular Biology, vol. 25, no. 5, pp. 1045-1050, 2005.

[29] A. J. G. Hanley, K. Williams, A. Festa, L. E. Wagenknecht, R. B. D'Agostino, and S. M. Haffner, "Liver markers and development of the metabolic syndrome: the insulin resistance atherosclerosis study," Diabetes, vol. 54, no. 11, pp. 3140-3147, 2005.

[30] M. L. Thakur, S. Sharma, A. Kumar et al., "Nonalcoholic fatty liver disease is associated with subclinical atherosclerosis independent of obesity and metabolic syndrome in Asian Indians," Atherosclerosis, vol. 223, no. 2, pp. 507-511, 2012.

[31] A. Kessler, Y. Levy, A. Roth et al., "Increased prevalence of NAFLD in patients with acute myocardial infarction independent of BMI," Hepatology, vol. 42, p. A623, 2005.

[32] M. Hamaguchi, T. Kojima, N. Takeda et al., "Nonalcoholic fatty liver disease is a novel predictor of cardiovascular disease," World Journal of Gastroenterology, vol. 13, no. 10, pp. 1579-1584, 2007.

[33] Y. Turan, "The nonalcoholic fatty liver disease fibrosis score is related to epicardial fat thickness and complexity of coronary artery disease," Angiology, vol. 71, no. 1, pp. 77-82, 2020. 